\title{
Resonator for microwave magnetoelectric effect
}

\author{
Vyacheslav Lobekin*, Alexandr Tatarenko, Andrei Belyshev, and Mirza Bichurin \\ The Yaroslav-the-Wise Novgorod State University, B.St.-Peterburgskaya str., 41, 173003, Veliky \\ Novgorod, Russia
}

\begin{abstract}
The design of a ring resonator made on a microstrip transmission line is presented. A study of ferromagnetic resonance using this design was made. Computer simulation of a microstrip ring resonator was carried out, and amplitude-frequency characteristics were obtained.
\end{abstract}

\section{Introduction}

A resonator is an important element of microwave integrated circuitry. A number of papers [1-3] are devoted to the study of various designs of resonators. Based on the phenomenon of resonance, it is possible to design various microwave devices: microwave filters [4,5], couplers [6], generators [7], antennas [8.9], etc. Resonators made on a microstrip transmission line are widely used. Microstrip transmission line is one of the main elements of microwave circuits and is used as components of microwave devices. To eliminate the end effect and increase the quality factor of the resonator, it is proposed to use a ring structure instead of a rectangular one. In this paper, we studied the design of a ring resonator made on a microstrip transmission line.

\section{The structure of a microstrip ring resonator}

The design of the microstrip ring resonator is shown in Fig. 1. The resonators are connected with the microstrip line due to regular strip lines diametrically connected to the ring resonator through the coupling gaps. The gap is chosen so that, on the one hand, the resonator field is not distorted, and on the other, the necessary coupling value is ensured. The initial width of the exciting microstrip lines is $\mathrm{W}=0.5 \mathrm{~mm}$, taking into account the input and output waveresistance of the line was $50 \mathrm{Ohms}$. The resonator is formed on a dielectric substrate with dimensions of $30 \times 24 \times 0,5 \mathrm{~mm}$, relative permittivity $\varepsilon=10$. The outer radius of the ring is $\mathrm{r} 1=14.3 \mathrm{~mm}$, the inner radius is $\mathrm{r} 2=11.3 \mathrm{~mm}$.

The resonant wavelength of the ring resonator is determined by the formula:

$$
\lambda_{p e 3}=\pi d_{c p} \sqrt{\varepsilon_{\ni \phi}}
$$

Average ring length:

$$
L=\lambda n
$$

* Corresponding author: slavalobekin@gmail.com 


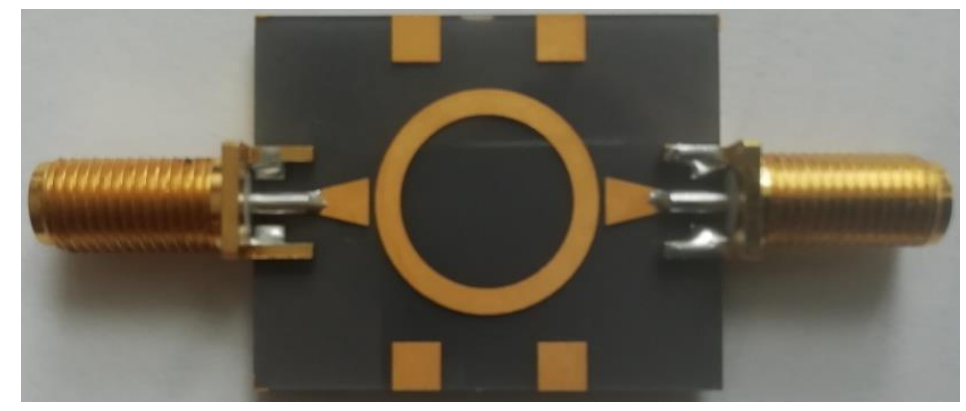

Fig. 1. The structure of microstrip ring resonator.

Where $\mathrm{n}$ is the number of wavelengths in the considered resonator, $\mathrm{n}=1,2,3$.

Loaded resonator quality factor:

$$
Q=\frac{f_{0}}{\Delta f}
$$

Where $\Delta \mathrm{f}$ is the width of the resonance curve, measured at a level of $3 \mathrm{~dB}$ from the maximum.

\section{Computer simulation of a microstrip ring resonator}

Computer modeling was carried out in the ANSYS HFFS program, which is the industry standard software for numerical electrodynamic modeling. The basic algorithm in HFSS is the three-dimensional finite element method (FEM), implemented in the frequency domain to calculate the behavior of electromagnetic fields on an arbitrary geometry with specified properties of materials.
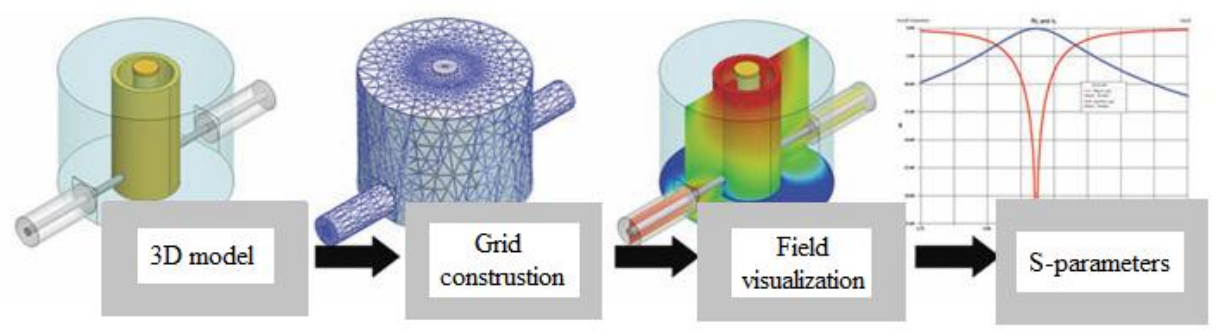

Fig. 2. Stages of work in HFSS.

Currently, the FEM has received deep theoretical justifications and is used to solve a very wide range of problems: stationary problems of heat distribution, diffusion, distribution of the electric field, other problems of field theory; hydromechanical problems, in particular, fluid flow in a porous medium; tasks of mechanics and strength, including design of airplanes, rockets and various spatial shells, etc. The basic concept of FEM is that any continuous quantity (temperature, pressure, displacement, etc.) can be approximated by a discrete model, which is built on a set of piecewise-continuous functions defined on a finite number of subdomains (elements). Piecewise continuous functions are determined using values of a continuous quantity in a finite number of points in the domain under consideration. 
When constructing a discrete model of continuous magnitude, one proceeds as follows: a finite number of points are fixed in the considered region. These points are called nodal or nodes. The value of a continuous quantity at each nodal point is considered a variable that must be determined. The domain of definition of a continuous quantity is divided into a finite number of subdomains called elements (or finite elements). These elements share common nodal points and together approximate the shape of the region. A continuous quantity is approximated on each element by a polynomial (or some other function), which is determined using the nodal values of this quantity. Each polynomial is determined for each element, but polynomials are selected in such a way as to maintain continuity of magnitude along the boundaries of the element. This polynomial is called the element function.

The HFSS program operation algorithm consists of the following stages: beginning solution type task - creating a model, including setting boundary conditions and an excitation source - setting a solver - solver cycle - presenting results (2D / 3D graphics, fields) - end.

ANSYS HFSS has the ability to:

- extract matrix parameters of the microwave structure (S, Y, Z - matrices);

— calculate the standing wave coefficient (SWR);

-obtain radiation and scattering parameters (radiation patterns, directional coefficients, realized antenna gain, EPR, etc.);

- display in 3D the distribution of currents, power flux density vectors, electromagnetic field distributions (in the near and far zones).

The proposed design of the ring resonator is used to increase the sensitivity of the measuring cell for the study of ferromagnetic resonance. In the narrower sense, FMR is the excitation of oscillations of the type of homogeneous (throughout the sample volume) precession of the magnetization vector $\mathrm{J}$ (spin waves with the wave vector $\mathrm{k}=0$ ), caused by a microwave microwave field $\mathrm{H}$, perpendicular to the constant magnetizing field $\mathrm{H} 0$. For this, a volume resonator was placed on the ring resonator $-\mathrm{D}=3 \mathrm{~mm}, \mathrm{~s}=0.5 \mathrm{~mm}$, consisting of a ferrite structure: a film of yttrium iron garnet (YIG) deposited on a gadolinium-gallium garnet (HHG) substrate and applied magnetic field H0=1500 Oe (Fig. 3) [10].

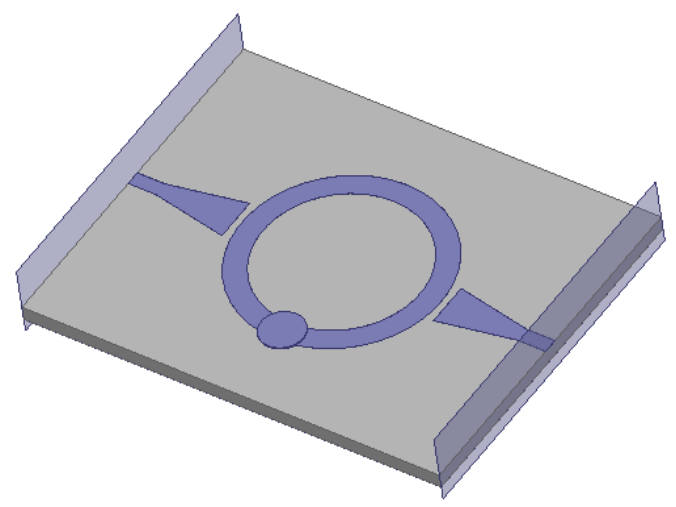

Fig. 3. 3D-model of a microstrip ring resonator with cavity resonator YIG-GGG.

Figure 4 shows the amplitude-frequency characteristic of a microstrip ring resonator and the amplitude-frequency characteristic with the YIG-GGG cavity resonator placed on a ring resonator. The obtained dependence shows that when the cavity resonator is placed on the ring, the attenuation increases compared to the usual design, -48.8 and $-33.9 \mathrm{~dB}$, respectively. This will allow you to choose the optimal parameters of the resonators for the design of magnetoelectric microwave devices, such as isolators. 


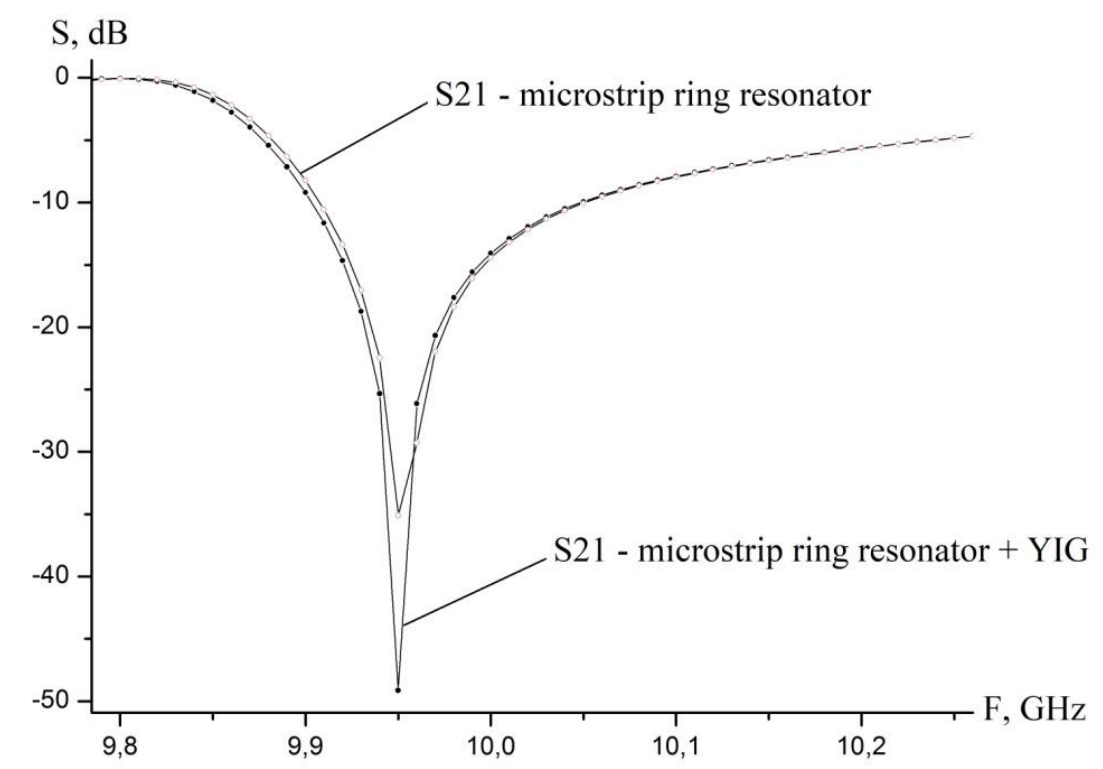

Fig. 4. Amplitude-frequency characteristic of a ferromagnetic resonance line.

\section{Conclusion}

The design of a microstrip ring resonator is investigated. Computer simulation of a ring resonator made on a microstrip transmission line was carried out in the ANSYS HFSS program. The obtained results of computer simulation allow us to choose the optimal resonator parameters for further experiments and modeling. A prototype microstrip ring resonator was made for further experiments.

This work was supported by the Russian Foundation for Basic Research (Project No. 19-07-00391).

\section{References}

1. E.I. Bocharov, M.A. Kondrashova, K.A. Rakova, E.Yu. Sedyshev, E.E. Tarasik, Elliptical ring resonators for microwave devices, VII All-Russian scientific and technical conference "Electronics and microwave microelectronics", p. 5 (2018)

2. Yu.V. Prokopenko, P.Yu. Sergienko, Electronics and communications. Electronic systems, №4, pp. 23-27 (2012)

3. A.A. Nkitin, A.B. Ustinov, A.A. Semenov, B.A. Kalinikos, Technical Physics (Zhurnal Tekhnicheskoi Fiziki), v. 82, №7, pp. 98-101 (2012)

4. A.S. Tatarenko, M.I. Bichurin, G. Srinivasan, Magnitoelektricheskie fil'truiushchie SVCh ustroistva. Vestnik NovGU. Ser. Tekhnicheskie nauki - Vestnik NovSU. Issue: Engineering Sciences, v. 26, pp. 168-172 (2004)

5. M.I. Bichurin, R.V. Petrov, A.S. Tatarenko, Magnitoelektricheskie SVCh ustroistva dlia FAR [Magnetoelectric microwave devices for phased array]. Sovremennye problemy nauki i obrazovaniia - Modern problems of science and education, v. 4 (2011)

6. A.N. Ostankov, N.N. Shchetinin, UHF and SHF Micro-strip Directional Coupler, Nauchno-prakticheskiy zhurnal Radiostroyeniye, v. 5, pp. 1-37 (2017) 
7. R.V. Petrov, N.A. Kolesnikov, M.I. Bichurin, Synchronous magnetoelectric generator, Patent of the Russian Federation № 2588986, 01.07.2016 (2016)

8. M.A. Khavanova, I.M. Yukhno, E.V. Kuzmin, V.N. Lobekin, R.V. Petrov, Research of characteristics of patch antenna based on magnetoelectric composite, Proceedings of the 28-th International Conference "Microwave\& Telecommunication Technology" (CriMiCo 2018), pp. 646-651 (2018)

9. M.A. Khavanova, V.A. Kamaikin, A.S. Tatarenko, R.V. Petrov, A. Porokhnyuk, T. Ueda, S. Tomita, Modeling of microwave antenna based on the multiferroic layered structure, Vestnik NovGU. Ser. Tekhnicheskie nauki - Vestnik NovSU. Issue: Engineering Sciences, v. 91, pp. 64-70 (2015)

10. S.N. Ivanov, M.I. Bichurin, G.A. Semenov, The technology of YIG-resonator forming for magnetoelectric microwave device, Vestnik NovGU. Ser. Tekhnicheskie nauki Vestnik NovSU. Issue: Engineering Sciences, v. 73, pp. 97-100 (2014) 